\title{
Optimal temporal-spatial PEV charging scheduling in active power distribution networks
}

\author{
Siyang Sun, Qiang Yang ${ }^{*}$ (D) and Wenjun Yan
}

\begin{abstract}
Background: The increasing penetration of a massive number of plug-in electric vehicles (PEVs) and distributed generators (DGs) into current power distribution networks imposes obvious challenges on power distribution network operation.

Methods: This paper presents an optimal temporal-spatial scheduling strategy of PEV charging demand in the presence of DGs. The solution is designed to ensure the reliable and secure operation of the active power distribution networks, the randomness introduced by PEVs and DGs can be managed through the appropriate scheduling of the PEV charging demand, as the PEVs can be considered as mobile energy storage units.
\end{abstract}

Results: As a result, the charging demands of PEVs are optimally scheduled temporally and spatially, which can improve the DG utilization efficiency as well as reduce the charging cost under real-time pricing (RTP).

Conclusions: The proposed scheduling strategy is evaluated through a series of simulations and the numerical results demonstrate the effectiveness and the benefits of the proposed solution.

Keywords: Plug-in electric vehicles (PEVs), Energy storage, Distribution generators (DGs), Charging demand, Charging scheduling strategy, Active power distribution networks, Real-time pricing (RTP)

\section{Background}

The growing concerns of fossil fuel consumption and greenhouse gas emission have motivated the quick development of plug-in electric vehicles (PEVs) and distributed generators (DGs). It is widely agreed that PEVs and DGs will become prevalent in recent decades, and high penetration level of PEVs and DGs is envisioned in future power distribution networks. For instance, it is anticipated that PEV will be a fairly large segment of US national fleet (at least $10 \%$ by 2020 and $50 \%$ by 2050 of new car sales projection) [1]. Moreover, the proportion of distributed renewable energy sources (e.g. photovoltaic and wind turbine) is reported to be $17.9 \%$ in total energy consumption of China in 2015, and the average annual growth rate reaches $9.42 \%$ [2].

On the one hand, the utilization of DGs can reduce the fossil fuel consumption and greenhouse gas

\footnotetext{
* Correspondence: qyang@zju.edu.cn

College of Electrical Engineering, Zhejiang University, 38 Zheda Rd., Hangzhou 310027, China
}

emission, and the large-scale deployment of PEVs can also promote the utilization of the renewable energy sources through the coordinated dispatching strategies of PEVs and renewable DGs. The benefits of the coordinated energy dispatch considering the availability of DGs and PEVs have been confirmed in some existing literature [3]. However, on the other hand, the uncoordinated integration of a massive number of PEVs and DGs into current active power distribution networks would bring direct challenges for network planning, control and management. The temporal and spatial characteristics of DG outputs and PEV charging demand impose a significant impact on load profiles of distribution networks, and hence results in unexpected voltage fluctuation and increased peak-valley gap [4]. In the presence of the increasing penetration of PEVs and DGs, a coordinated scheduling strategy for PEV charging demand and DG outputs management needs to be adopted in current active power distribution networks to ensure the reliable and economical network operation. 
In literature, a collection of research effort has been made to address the challenges of the optimal scheduling strategies of PEV charging (e.g. [5-13]), but only a few works considered the interaction with DGs (e.g. [3, 14-17]). The authors in [3] exploited three coordinated wind-PEV energy dispatching approaches (i.e., valley searching, interruptible and variable-rate energy dispatching), aiming to optimize the utilization efficiency of wind power generation as well as meet the dynamic power demands. In [14], the self-scheduling problem of an aggregator of PEVs purchasing energy in the dayahead market and offering balancing services for a wind power producer was discussed using a probabilistic virtual battery model and a scenario-based robust approach. Moreover, a two-level architecture based on model predictive control was presented to deal with distributed energy resources, renewables and storage devices in microgrids with less computational complexity in [15]. The authors in [16] proposed a PEV charging policy that considers every 5 min real-time market energy price signal to increase the penetration of distributed PV solar arrays. Last but not least, a charging process undergone for PEVs in parking lot areas using PV-based charging facilities was presented to evaluate the voltage profiles, peak demand and charging cost of this scenario in [17].

However, the aforementioned solutions have some obvious limitations. Firstly, it has been reported that current PEVs can be charged either in normal charging mode at home, or in fast charging mode at fast charging stations [18], and the two charging modes have totally different characteristics, which has not been considered in the related works. A comprehensive scheduling strategy needs to be proposed including two charging modes. Furthermore, most of the literature only aimed to solve either the reliable operation of the distribution networks or the economical charging of PEV owners. However, both aspects should be focused on to achieve a win-win result. In addition, to the authors' best knowledge, most of the related works are based on the day-ahead prediction of DG outputs, there still lacks of PEV charging scheduling strategies to ensure the real-time performance.

To this end, this paper addressed the challenges of PEV charging scheduling strategy and presented a novel temporal-spatial optimal scheduling strategy of PEV charging demand considering the existence of DGs. The main technical contributions made in this work are as follows: (1) PEV charging demand is scheduled separately based on the characteristics of two charging modes with DG outputs and real-time pricing (RTP), spatially scheduling for fast charging demand and temporally scheduling for normal charging demand; (2) the objective function of the proposed temporal-spatial scheduling strategy considers both the reliable and economical network operation, trying to improve the utilization efficiency of DGs as well as reduce the cost of PEV charging considering RTP.

The remainder of this paper is organized as follows: Section 2 introduces the characteristics of two PEV charging modes, and presents the framework of the temporal-spatial scheduling strategy; Section 3 presents the mathematical formulation of the temporal-spatial scheduling strategy; Section 4 evaluates the benefits of the temporal-spatial scheduling strategy through a set of simulations with field data; finally, a set of conclusive remarks are given in Section 5.

\section{The framework of the temporal-spatial schedul- ing strategy}

Currently, two PEV charging modes are widely adopted: normal charging mode at home or parking lots with lower charging power level, and fast charging mode at fast charging stations with higher charging power level, the typical charging duration is $20-30 \mathrm{~min}$ [18]. Besides the charging power level, the characteristics of such two charging modes are also intrinsically different. When a $\mathrm{PEV}$ is driving to the destination but the $\mathrm{SoC}$ is too low to complete the rest trip, it should find a fast charging station to get charged with the fast charging mode as soon as possible. However, the normal charging often happens when a PEV arrives at home or workplaces and it doesn't hurry to get fully charged $[19,20]$.

Therefore, it is obvious that the PEV fast charging demand is unpostponable and uninterruptible, but the PEVs with fast charging demand can be scheduled to get charged in an optimal fast charging station. The fast charging demand of PEVs can be considered spatially schedulable but temporally unschedulable. On the contrary, the normal charging demand is interruptible, which can be shifted over time, but the charging place is totally fixed. The normal charging time can be scheduled to consume the DGs optimally with minimal cost, which means the normal charging demand can be considered temporally schedulable but spatially unschedulable.

The differences of these two PEV charging modes are illustrated in detailed in Table 1.

Because of the totally different characteristics of two PEV charging modes, the PEV charging scheduling strategy should consider two types of the PEV charging demand separately. The framework of the proposed

Table 1 The characteristics of two PEV charging modes

\begin{tabular}{lll}
\hline & fast charging & normal charging \\
\hline charging power level & higher & lower \\
charging place & fast charging stations & home or workplaces \\
space characteristics & schedulable & unschedulable \\
time characteristics & unschedulable & schedulable \\
\hline
\end{tabular}


temporal-spatial scheduling strategy of PEV charging is shown in Fig. 1. As mentioned before, the normal charging demand is scheduled temporally and the fast charging demand is scheduled spatially, in order to achieve the optimal interaction with DGs and grids.

The proposed temporal-spatial scheduling solution aims to meet the PEV charging demand with minimal cost as well as optimize the DG utilization efficiency both temporally and spatially. PEVs can be assumed as mobile energy storage units which are considered with more flexible and less economical cost compared with the traditional battery energy storage.

\section{The formulation of the temporal-spatial sched- uling strategy}

Based on the characteristics of two PEV charging modes illustrated in Section 2, the temporal-spatial scheduling strategy separately schedules PEV normal charging demand and fast charging demand temporally and spatially.

\subsection{Spatial scheduling strategy of fast charging}

The spatial scheduling strategy aims to spatially schedule the PEVs with fast charging demand to get charged at optimal fast charging stations to consume the outputs of DGs with minimal cost. The strategy spatially schedules the PEV fast charging based on the fast charging requests $R_{f c h}$ and the profiles of the DGs and the distribution network, as illustrated in Fig. 2. In this work, a set of assumptions are made as follows:
(1) There assumes to be a geographical area with several PEV fast charging stations in the distribution network, and each charging station is assumed to be connected by a DG;

(2) The whole day is divided into 48 time slots (i.e. 30 min per slot) $[19,20]$, and the output of DGs, the demand of baseload, and RTP are assumed to be detectable at the start of each time slot and remain unchanged during each time slot;

(3) The PEVs are assumed to keep driving if the $S o C$ is more than 0.3. When the $S o C$ is lower than 0.3 when the current time slot ends, the PEV needs to get charged in fast charging mode in the next time slot;

(4) There assumes to be an aggregation center with the access to the $S o C$ and the location information of each PEV with the message format of $R_{f c h}$ (SoC, location), to schedule the PEV fast charging behaviors at the start of each time slots.

The objective function $F_{f c h}$ aims to improve the utilization efficiency of DGs, at the meanwhile reduce the cost of charging, as shown in (1):

$$
\min F_{f c h}=\min \left(\omega_{1} \sum_{i=1}^{m}\left|P_{b a s e}^{i}+P_{f c h}^{i}-P_{D G}^{i}\right|+\omega_{2} \frac{\sum_{j-1}^{n} \cos t^{j}}{n}\right)
$$

where

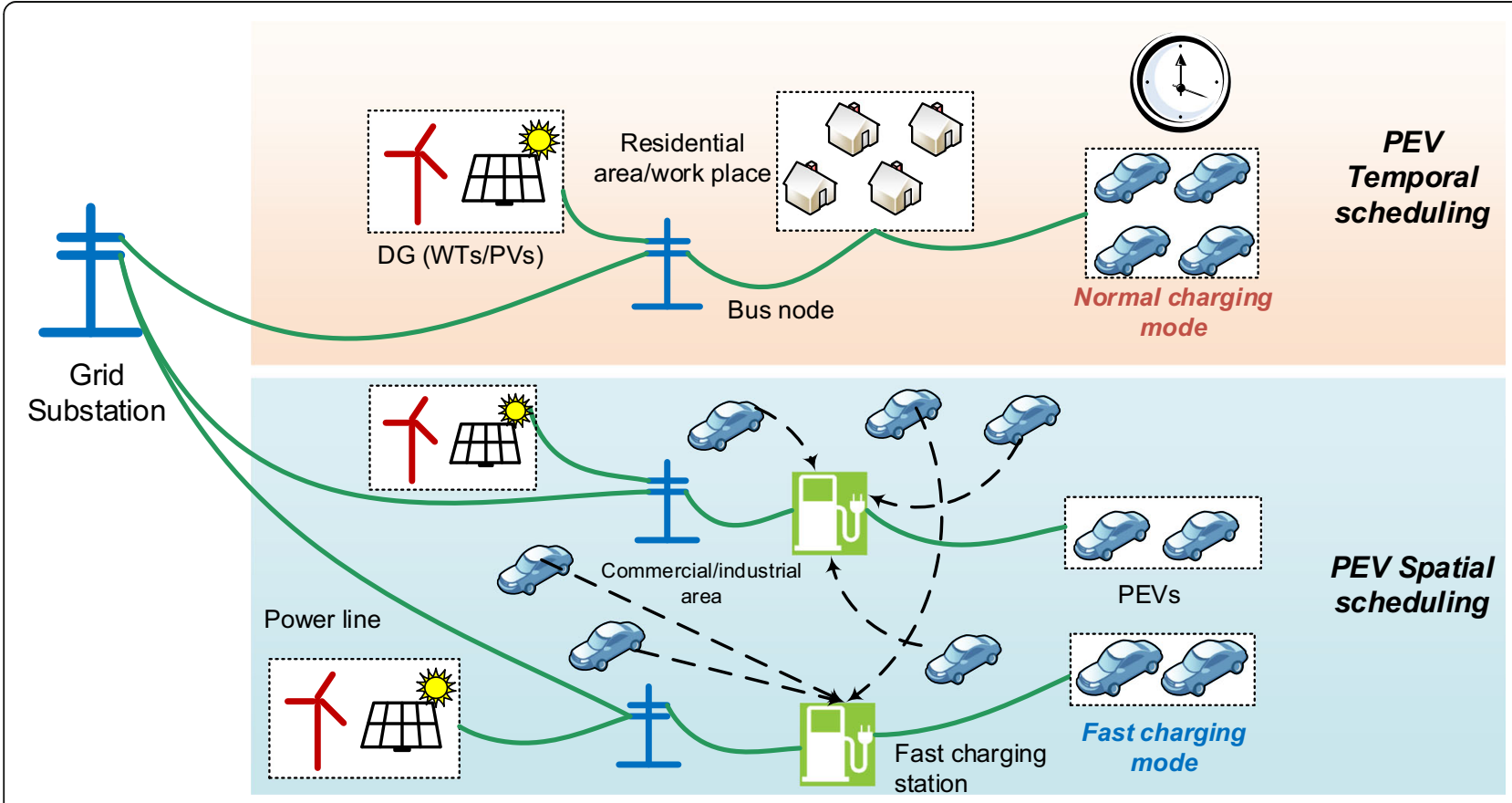

Fig. 1 The framework of the temporal-spatial scheduling strategy 


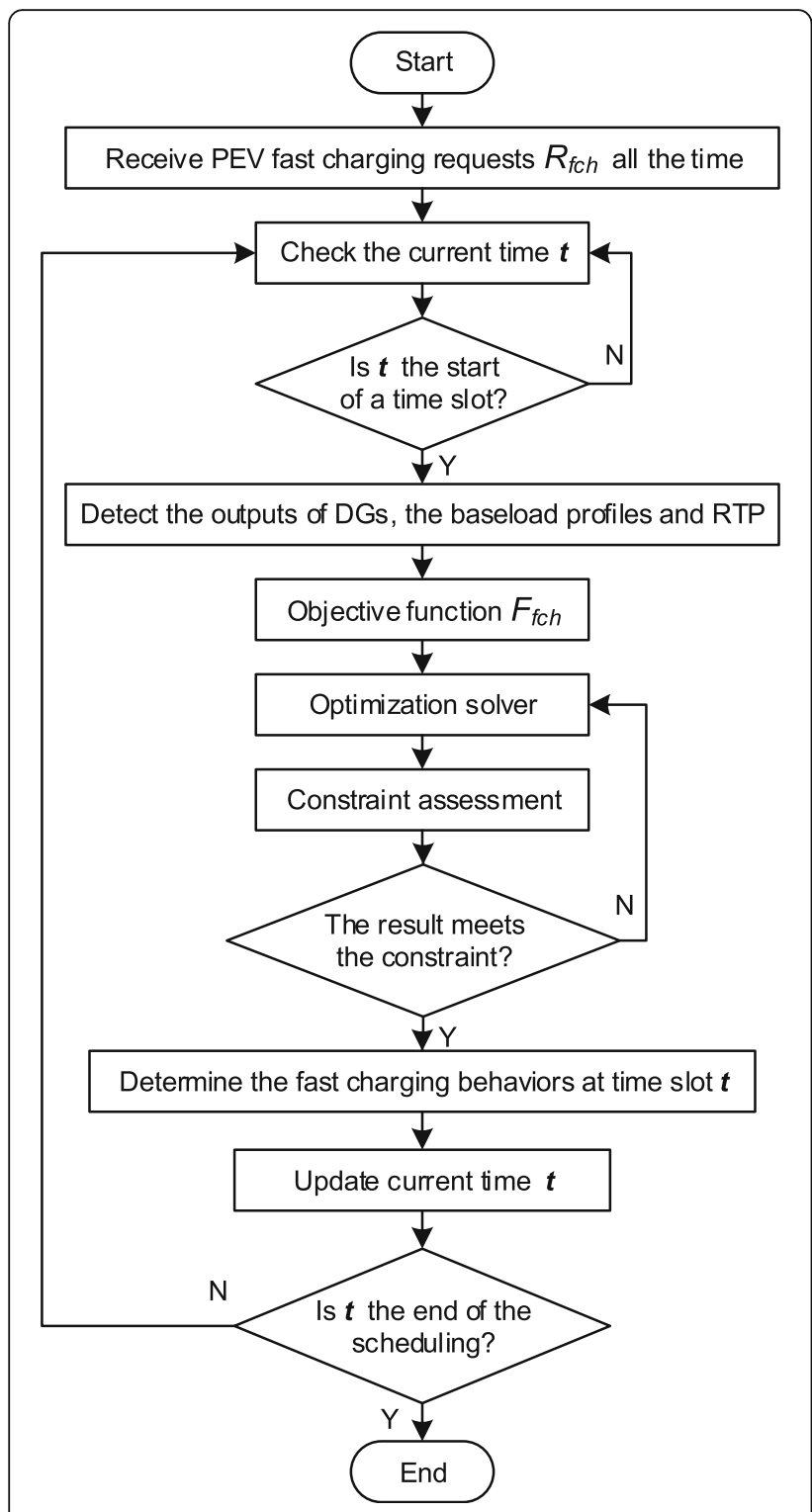

Fig. 2 The flowchart of spatial scheduling strategy of fast charging

$$
\operatorname{cost}=\left(1-\mathrm{SoC}_{0}\right) \cdot C_{b a t} \cdot R T P+\operatorname{dis} \cdot E_{c} \cdot R T P
$$

$\omega_{1}$ and $\omega_{2}$ e assigned a value according to the different situation.

The first term in the objective function $F_{f c h}$ represents the sum of the active power in each station, to make sure that the electric energy generated by DGs can be consumed spatially by PEVs as much as possible; the second term represents the average charging cost of each PEV. The total charging cost also consists of two parts, as shown in (2). The first part is the charging cost to fully charge the battery, and the second part represents the scheduling cost (i.e. the distance to the objective station). The variables in this objective function are the different charging places for each PEV with fast charging demand.

The objective function is subject to:

$$
\begin{aligned}
& \left|\Delta U^{k}\right|<0.1 \cdot U_{0}, \forall k=1,2, \cdots, l \\
& \operatorname{dis}<\frac{S_{o} C_{0} \cdot C_{b a t}}{E_{c}}
\end{aligned}
$$

where (3) means the voltage fluctuation on bus node $k$ cannot exceed the upper and lower bounds of the node voltage; and (4) means that the PEVs cannot be scheduled to a charging station farther than the remaining mileage.

The proposed spatial scheduling strategy of PEV fast charging demand is carried out at the start of each time slot. Once the fast charging behaviors of all PEVs are determined by the scheduling strategy, the PEVs with fast charging demand will get charged in the assigned charging stations. Likely, in the next time slot, the DG output generation, the baseline demand, and RTP can be accurately detected again. Thus, the spatial scheduling strategy will repeat in every time slot with real-time information, which can ensure the real-time performance of the scheduling strategy.

Through the proposed spatial scheduling strategy of PEV fast charging demand, the electric power generated by DGs can be scheduled spatially to be consumed by PEV fast charging demand as much as possible with minimal cost. Moreover, the active power on every bus node in the distribution network will be more balanced.

\subsection{Temporal scheduling strategy of normal charging}

Unlike the PEV spatial scheduling, the temporal scheduling strategy aims to temporally schedule the PEVs with normal charging demand to get charged in optimal times to consume the distributed generation with minimal cost. The strategy schedules the PEV normal charging demand based on the normal charging requests $R_{n c h}$, the outputs of DGs, and the baseload profiles of the charging bus node, as illustrated in Fig. 3.

Here, the following assumptions are made:

(1) The whole day is divided into 48 time slots (i.e. 30 min per slot), and the output of DGs, the demand of baseload, and RTP are assumed to be predictable day-ahead and remain unchanged during each time slot;

(2) There is assumed to be an aggregation center able to communicate with each PEV, schedule the normal charging behaviors and estimate PEV normal charging demand in the future;

(3) When a PEV arrives at home, if the $S o C$ is less than 0.8 , it is assumed to have a normal charging request to get fully charged at night; 


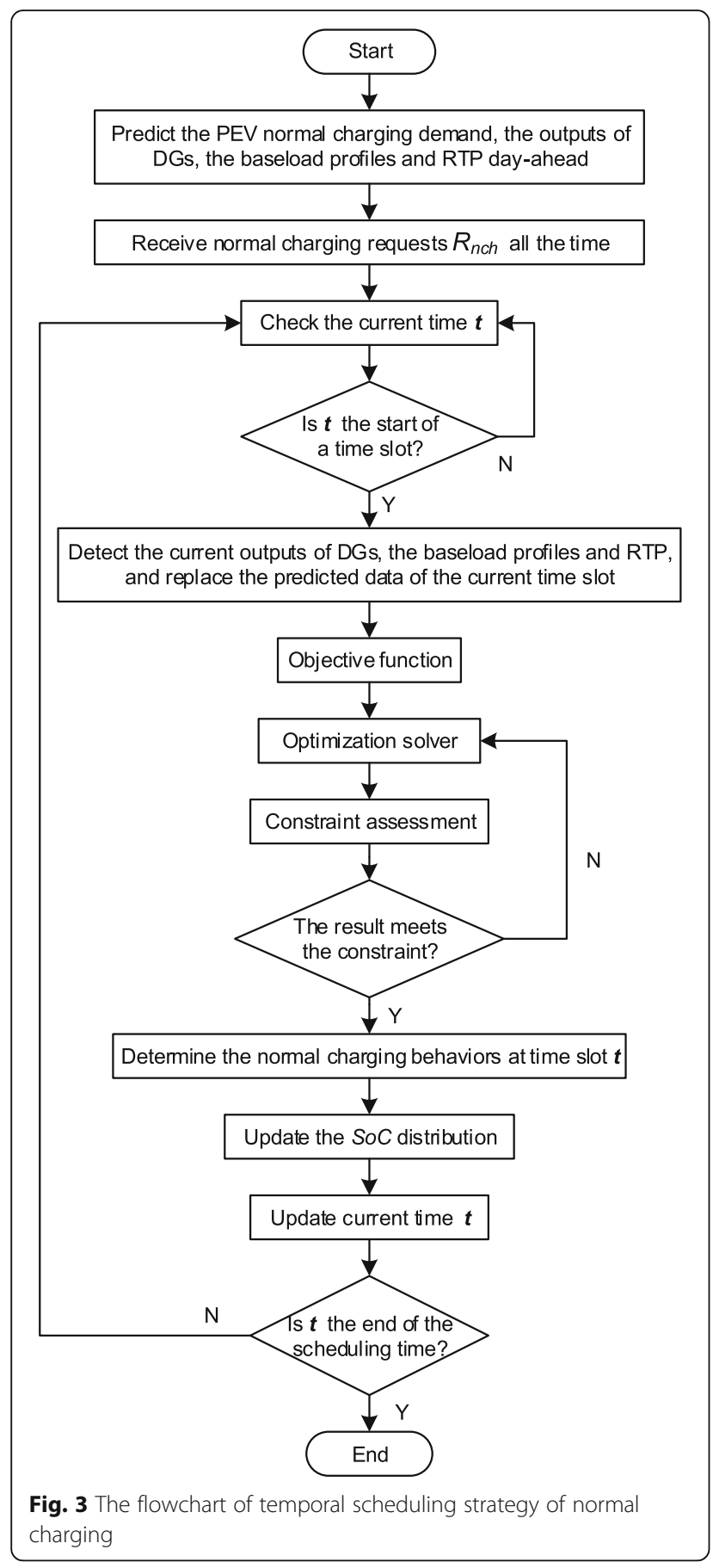

(4) PEV sends a message to the aggregation center when it arrives at home, including the information of arrival time, current $S o C$, battery capacity, and leaving time, with the format of $R_{n c h}\left(t_{a}, S o C_{0}, C_{b a t}, t_{\text {end }}\right)$.

The objective function $F_{n c h}$ also aims to improve the utilization efficiency of DGs, at the meanwhile reduce the cost of charging, as shown in (5):

$$
\min F_{n c h}=\min \left(\omega_{1} \sum_{t=1}^{48}\left(\left|P_{\text {base }}^{t}+P_{n c h}^{t}-P_{D G}^{t}\right|+\omega_{2} \sum_{j=1}^{n} \cos t_{j}^{t}\right)\right)
$$

where

$$
\sum_{j=1}^{n} \operatorname{cost}_{j}=P_{n c h} \cdot R T P \cdot T
$$

$\omega_{1}$ and $\omega_{2}$ are coefficient factors, which can be assigned a value according to the different situation.

The first term in the objective function $F_{n c h}$ represents the active power on a certain bus in 48 time slots to make sure that the electric energy generated by DGs can be consumed temporally by PEVs as much as possible; the second term represents the total charging cost in each time slot, as shown in (6). As the time slot is $30 \mathrm{~min}$ long, $T$ equals 0.5 . The variables in this objective function are the different charging time slots for the PEVs with normal charging demand.

The objective function is subject to:

$$
\begin{aligned}
& \left|\Delta U_{t}^{k}\right|<0.1 \cdot U_{0}, \forall t=1,2, \cdots, 48, \forall k=1,2, \cdots, l \\
& \text { So } C^{t_{\text {end }}}=1
\end{aligned}
$$

where $\left|\Delta U_{t}^{k}\right|$ in (7) represents the voltage fluctuation on bus node $k$ at time slot $t$, which means the voltage fluctuation of each bus node in each time slot cannot exceed the upper and lower bounds of the node voltage; and (8) means that the battery should be fully charged at the leaving time $t_{\text {end }}$. It should be noticed about the constraint (7) that although the proposed temporal scheduling strategy of normal charging focuses on the fixed charging node, the influence on the distribution network still needs to be considered.

As the temporal profiles of distribution networks in the future are needed in the proposed temporal scheduling strategy, the outputs of DGs, the demand of baseload, and RTP should be predicted day-ahead, and the predicting algorithm is available in [21]. Moreover, the temporal PEV normal charging demand in the future is also needed to be estimated, which can be obtained from the modeling approach in $[19,20]$ Once the normal charging behaviors in the current time slot of all PEVs

Table 2 PEV battery capacity parameters

\begin{tabular}{lll}
\hline battery type & $M 1$ & $N 1$ \\
\hline$C_{\text {bat }}(k W h)$ & $\mu_{C_{b a t}}^{M 1}=28.5 \sigma_{c_{b a t}}^{M 1}=14.7$ & $\mu_{C_{b a t} 1}^{N 1}=23.0 \sigma_{C_{b a t}}^{N 1}=9.5$ \\
& $C_{\max }^{M 1}=72.0 C_{\min }^{M 1}=10.0$ & $C_{\max }^{N 1}=40.0 C_{\min }^{N 1}=9.6$ \\
$E_{C}(k W h / k m)$ & $E_{c}^{M 1}=0.159$ & $E_{c}^{N 1}=0.185$ \\
Market share $m s$ & $m s^{M 1}=89 \%$ & $m s^{N 1}=11 \%$ \\
\hline
\end{tabular}




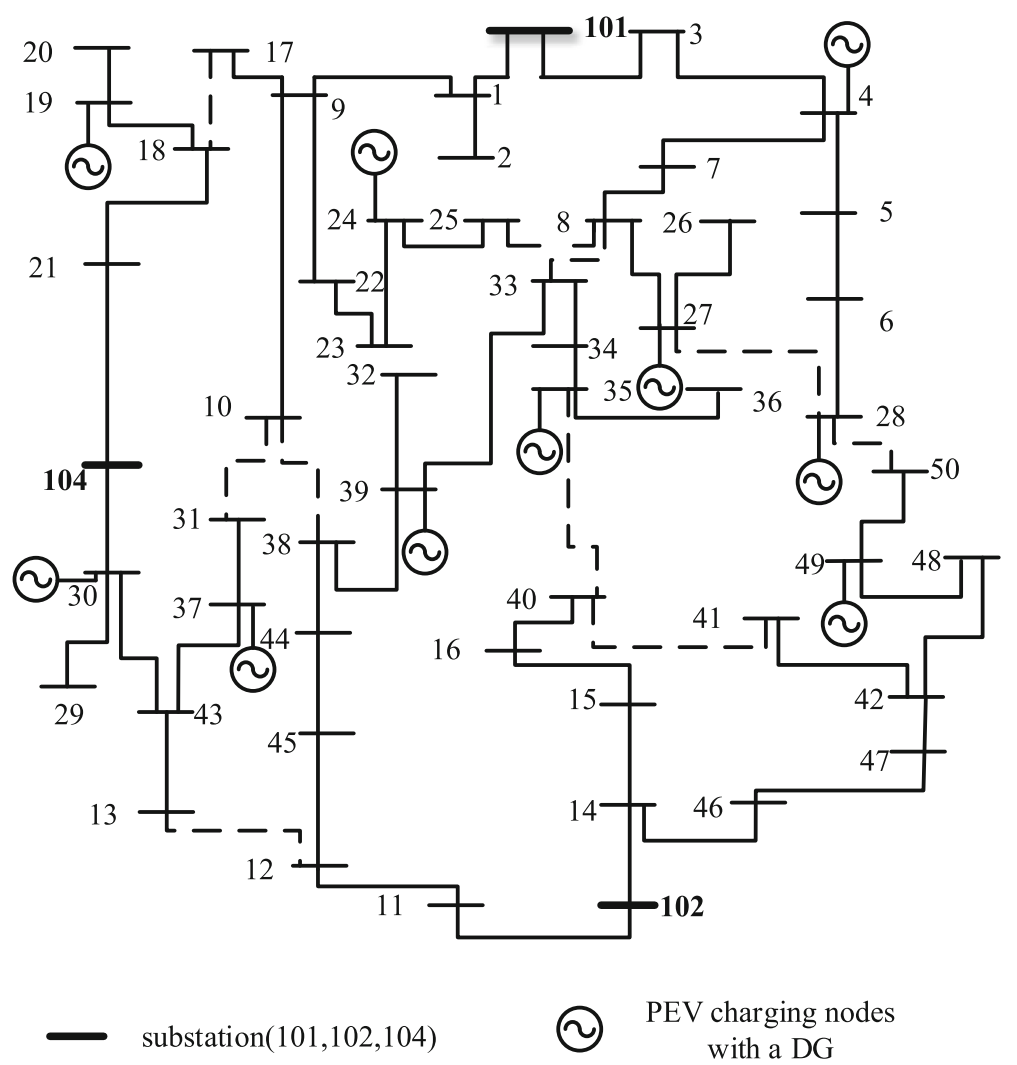

Fig. 4 The adopted IEEE 53-bus test feeder

are determined by the strategy, the $S o C$ distribution (i.e. the expected charging duration) of PEVs with normal charging demand in the next time slot can be updated, and the real-time output of DGs, the baseload profiles and the RTP can also be detected to replace the predicted data. Thus, the temporal scheduling strategy will repeat in each time slot, which can ensure the real-time performance and the accuracy of the scheduling strategy.

Through the proposed temporal scheduling strategy of PEV normal charging, the electric power generated by DGs can get consumed by PEV normal charging demand as much as possible with minimal cost in every time slot. Moreover, the active power on the charging bus node in every time slot will also be more balanced.

In a word, based on the proposed temporal-spatial scheduling strategy, the PEV charging demand can be separately scheduled to consume the electric power generated by DGs with minimal cost temporally and spatially, and the operation of the distribution networks can become more reliable and economical.

\section{Case studies and simulation results}

This section carries out a set of case studies to implement and justify the effectiveness of the proposed solution.

\subsection{Statistics and assumptions}

In this work, normal charging mode and fast charging mode are both adopted with the typical charging power $P_{n-c h}$ and $P_{f-c h}$ of $3.3 \mathrm{~kW}(220 \mathrm{~V} / 15 \mathrm{~A})$ and $50 \mathrm{~kW}$ (400 V/125A), respectively [22]. In addition, the battery capacity of typical PEVs can be obtained from [23], which follows the normal distribution $N\left(\mu, \sigma^{2}\right)$, as shown in Table 2.

The IEEE 53-bus test feeder is adopted in this work to further demonstrate the effectiveness of the proposed temporal-spatial scheduling strategy of PEV charging [24]. The typical daily baseload curve in Beijing are obtained from [25] and scaled down proportionally. As

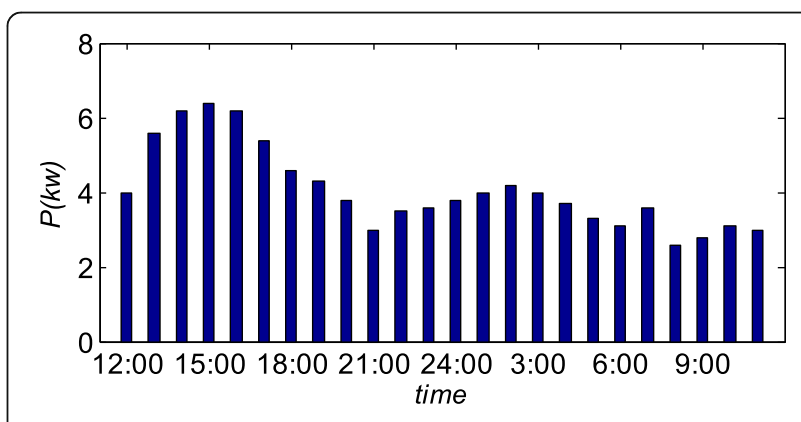

Fig. 5 The typical curve of DG output 


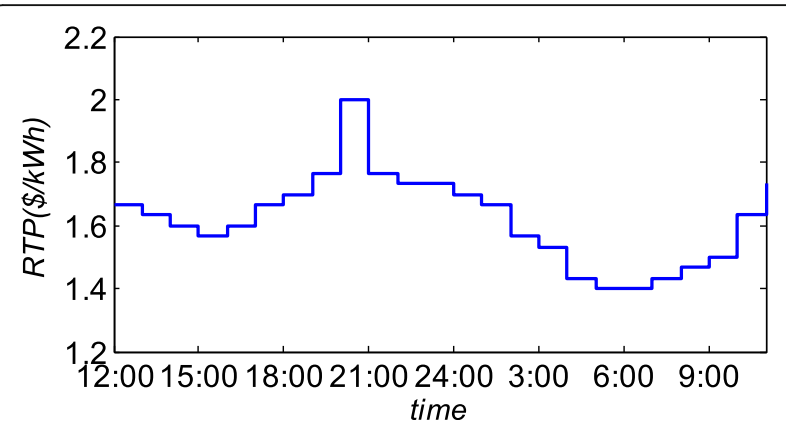

Fig. 6 The adopted RTP scheme in simulations

is shown in Fig. 4, there assumes to be 10 PEV charging nodes in the distribution network, and each node is connected by a DG, which is assumed to be a combination of photovoltaic (PV) and wind turbine, with the typical daily curve of output shown in Fig. 5, which can be predicted day-ahead [26, 27]. In addition, the typical daily curve of RTP is shown in Fig. 6, which is also assumed to be day-ahead predictable [28].

Furthermore, the PEV home arrival time can be obtained from National Household Travel Survey (NHTS) [29], and the normal charging demand is estimated based on the modeling approach in $[19,20]$. It is assumed that there are in total $n_{f c h}=10^{4}$ PEVs in this geographical area in fast charging scenario and there are $n_{n c h}=500 \mathrm{PEVs}$ on each bus node to get normal charged in normal charging scenario. The initial $\mathrm{SoC}$ of two scheduling scenarios are both assumed to follow the normal distribution $N\left(\mu, \sigma^{2}\right)$ with the mean value $\mu$ and the standard deviation $\sigma$ of 0.5 and 0.1. In addition, the distance from the PEV current location to each charging station is assumed to follow the normal distribution $N\left(15,4^{2}\right)$.

\subsection{Spatial scheduling strategy of fast charging}

The proposed spatial scheduling strategy is applied in a geographical area, such as the adopted IEEE 53-bus test feeder. It should be noticed that the spatial scheduling strategy in each time slot is independent from each other. The scenario at 17:00 is carried out for an example as follows.

Case 1: uncoordinated charging strategy.

In the uncoordinated charging strategy, the charging behaviors are totally uncontrolled. Thus all PEVs with fast charging requests will get charged in the nearest charging station, as shown in (9),

$$
d i s^{j}=\text { mindis }
$$

where dis is the vector of the distances dis from the PEV current location to each charging station.

In this case, the function (1) can be easily calculated.

Case 2: the proposed spatial scheduling strategy.

PEVs with fast charging demand are scheduled to the optimal charging stations according to the proposed spatial scheduling strategy, as illustrated in Section 3.1. The objective function (1) can be solved using YALMIP optimization toolbox in MATLAB. Moreover, the optimization result is also verified by MATPOWER6.0 in MATLAB, in order to ensure the result satisfies the voltage fluctuation constraint (3). If the result exceeds the voltage constraint, the optimization process will repeat again to find the next optimal result that satisfies the voltage fluctuation constraint.

The numerical results for two different cases are presented in Table 3. It can be observed that the proposed spatial scheduling strategy can significantly improve the utilization efficiency of DGs with a little cost increase. The charging cost increases because the PEV will be scheduled to a farther charging station to consume the output of DGs. However, the increase of cost is too small, which can be ignored according to the results in Table 3.

In order to further demonstrate the benefits of the proposed spatial scheduling strategy, the utilization efficiency of DGs in two cases are shown in Fig. 7, and the active power flow from/to the grid in each charging station is shown in Fig. 8. It can be seen that the utilization efficiency of all DGs is improved and the active power on the bus nodes of each charging station becomes more spatially balanced.

In addition, Table 3 provides the standard deviation of the bus voltage in the test network. It is obvious that the voltage profiles also become better by the proposed spatial scheduling strategy compared with uncoordinated case, which will be more effective when the penetration of PEVs and DGs gets larger.

\subsection{Temporal scheduling strategy of normal charging}

The proposed temporal scheduling strategy is applied in fixed bus nodes of the distribution network, as shown in Fig. 4. In this work, there are also two cases in temporal scheduling strategy for PEV normal charging mode.

Case 1: uncoordinated charging strategy.

Table 3 The numerical results of two evaluated cases

\begin{tabular}{lllll}
\hline Different cases & Objective function & First term & Second term & Voltage standard deviation \\
\hline without PEV & - & - & - & 0.0065 \\
Case 1 & 7.001 & 4.710 & 2.291 & 0.0085 \\
Case 2 & 3.270 & 0.976 & 2.294 & 0.0072 \\
\hline
\end{tabular}




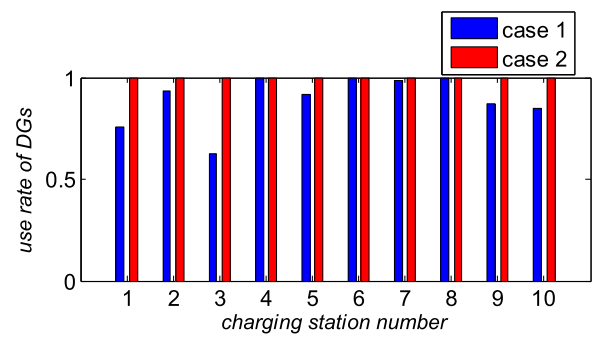

Fig. 7 DG utilization efficiency (fast charging)

In the uncoordinated charging strategy, the charging behaviors are totally uncontrolled. All PEVs will start normal charging as soon as they arrive at home, as shown in (10). Moreover, the normal charging is also uninterrupted until the battery is fully charged.

$$
t_{s c}=t_{a}
$$

Case 2: the proposed temporal scheduling strategy.

As is mentioned in Section 3.2, the objective function (5) can be also solved using YALMIP optimization toolbox in MATLAB. As same as Section 4.2, the optimization result is also verified by MATPOWER6.0 in MATLAB, in order to ensure the result satisfies the voltage fluctuation constraint (7). If the result exceeds the voltage constraint, the optimization process will be repeated again to identify the eligible solution.

The results of two cases are shown in Table 4. It can be observed that the proposed temporal scheduling strategy can significantly improve the utilization efficiency of DGs and reduce the charging cost.

In order to further demonstrate the benefits of the proposed temporal scheduling strategy, the utilization efficiency of DGs in two cases are shown in Fig. 9. Through the proposed temporal scheduling strategy, the PEV normal charging can avoid the peak time of RTP (i.e. 18:00 pm-20:00 pm), and the utilization efficiency of DGs in most of time slots (i.e. 24:00 pm-6:00 am) is significantly improved. In addition, the active power flow from/to the grid during a day is shown in Fig. 10. The

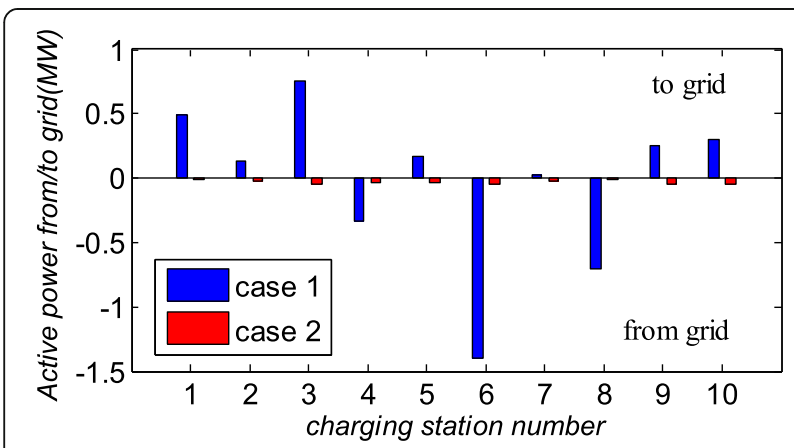

Fig. 8 The active power from/to the grid in each station
Table 4 The results of two cases

\begin{tabular}{llll}
\hline Different cases & Objective function & First term & Second term \\
\hline Case 1 & 46.09 & 29.09 & 17.00 \\
Case 2 & 35.88 & 23.86 & 12.03 \\
\hline
\end{tabular}

fluctuation of the active power flow becomes more balanced during a day through the proposed temporal scheduling strategy. It should be noted that at 17:00 pm-20:00 pm, the PEV normal charging demand is scheduled to other time to avoid the peak hours of RTP.

\section{Conclusions and future work}

This paper presented a novel temporal-spatial optimal scheduling strategy of PEV charging demand with the existence of DGs. PEV is assumed to be excellent mobile energy storage to optimally consume the distributed generation temporally and spatially. The PEV charging behaviors are separately scheduled temporally and spatially based on the characteristics of two PEV charging modes, normal charging and fast charging. The objective function aims to improve the utilization efficiency of DGs, at the meanwhile reduce the cost of charging under RTP. The proposed scheduling strategy is further carried out for a series of case studies with field data and reasonable assumptions. The results demonstrate the benefits of the proposed strategy considering the large-scale integration of PEVs and DGs.

In the future, a number of directions are considered worth further research efforts. The temporal-spatial strategy can be extended to schedule the two PEV charging behaviors simultaneously; the traffic networks can also be adopted into the spatial scheduling strategy to obtain accurate distance information; and the scheduling of PEV normal charging demand can be further developed as an intelligent house system with some certain household applications. The research outcome will be provided in future publications.

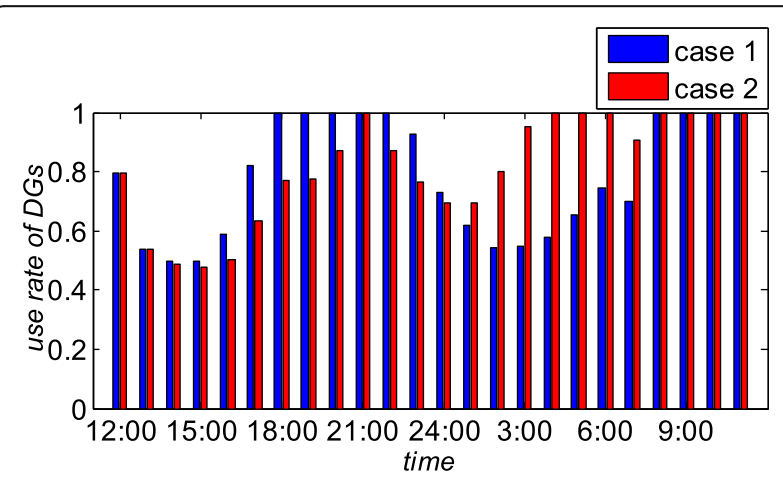

Fig. 9 DG utilization efficiency (normal charging) 


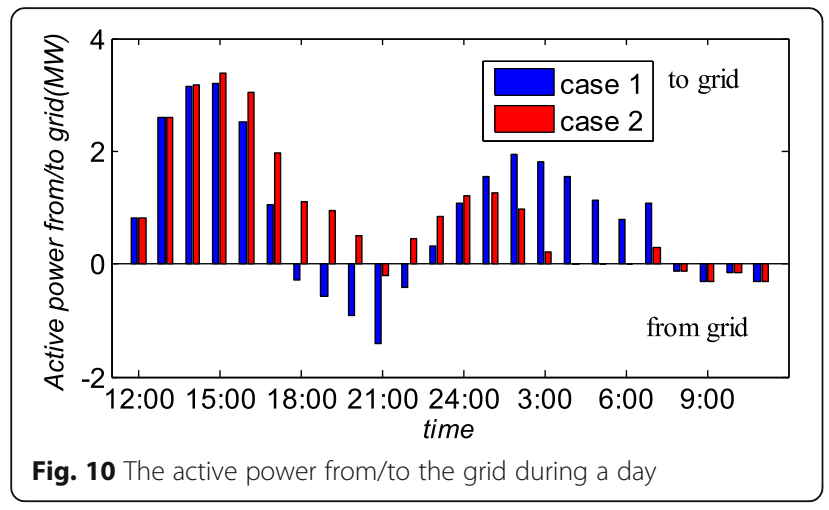

\section{Nomenclature}

\subsection{PEV related variables}

$C_{\text {bat }}$ PEV battery capacity. cost PEV charging cost.

dis Distance to a charging station of a PEV.

$E_{c}$ Energy consumption per kilometer of battery. $m s$ Market share of each type of PEVs.

$n$ Number of PEVs.

$P_{f c h}$ PEV fast charging demand.

$P_{f-c h}$ Fast charging power.

$P_{n c h}$ PEV normal charging demand.

$P_{n-c h}$ Normal charging power.

$R_{f c h}$ Message format of fast charging.

$R_{n c h}$ Message format of normal charging.

$\mathrm{SoC}_{0}$ Initial $\mathrm{SoC}$ of charging.

$t_{a}$ Home arrival time.

$t_{\text {end }}$ Leaving time.

$t_{s c}$ Charging start time.

\subsection{DG and grid related variables}

$i$ Number of fast charging stations.

$k$ Number of bus nodes.

$l$ Maximum number of bus nodes.

$m$ Maximum number of fast charging stations.

$P_{\text {base }}$ Baseload demand.

$P_{D G}$ Output of DGs.

RTP Real-time pricing.

$U_{0}$ Standard node voltage.

$\Delta U$ Voltage fluctuation.

$\omega_{1}$ Coefficient factor.

$\omega_{2}$ Coefficient factor.

\section{Acknowledgements}

This work is supported by in part by The National Key Research and Development Program of China (Basic Research Class 2017YFB0903000) -Basic Theories and Methods of Analysis and Control of the Cyber Physical Systems for Power Grid, and the Natural Science Foundation of Zhejiang Province (LZ15E070001)

\section{Authors' contributions}

QY conceived and designed the study. QY and SS performed the experiments. QY and SS wrote the paper. QY, SS and WY reviewed and edited the manuscript. All authors read and approve the manuscript.

\section{Competing interests}

The authors declare that they have no competing interests.

Received: 3 June 2017 Accepted: 8 September 2017

Published online: 19 September 2017

\section{References}

1. Mukherjee, J. C., \& Gupta, A. (Jan. 2017). Distributed charge scheduling of plug-in electric vehicles using inter-aggregator collaboration. IEEE Transactions on Smart Grid, 8(1), 331-341.

2. National Bureau of Statistics of the People's Republic of China, "Statistical communique on national economic and social development in 2015", Available [Online]: http://www.stats.gov.cn/tjsj/zxfb/201602/t20160229 1323991.html, Feb. 2016

3. Wu, T., Yang, Q., Bao, Z., \& Yan, W. (Sept. 2013). Coordinated energy dispatching in microgrid with wind power generation and plug-in electric vehicles. IEEE Trans Smart Grid, 4(3), 1453-1463.

4. Yao, W., Chung, C. Y., Wen, F., Qin, M., \& Xue, Y. (Jan. 2016). Scenario-based comprehensive expansion planning for distribution systems considering integration of plug-in electric vehicles. IEEE Trans Power Syst, 31(1), 317-328.

5. Alahyari, A., Fotuhi-Firuzabad, M., \& Rastegar, M. (July 2015). Incorporating customer reliability cost in PEV charge scheduling schemes considering vehicle-to-home capability. IEEE Trans Veh Technol, 64(7), 2783-2791.

6. Tang, W., Bi, S., \& Zhang, Y. J. (Nov. 2014). Online coordinated charging decision algorithm for electric vehicles without future information. IEEE Transactions on Smart Grid, 5(6), 2810-2824.

7. Xu, Z., Su, W., Hu, Z., Song, Y., \& Zhang, H. (Jan. 2016). A hierarchical framework for coordinated charging of plug-in electric vehicles in China. IEEE Transactions on Smart Grid, 7(1), 428-438.

8. March 2017). A model predictive control approach for low-complexity electric vehicle charging scheduling: Optimality and scalability. IEEE Trans Power Syst, 32(2), 1050-1063.W. Tang and Y. J. (Angela) Zhang, "A model predictive control approach for low-complexity electric vehicle charging scheduling: Optimality and scalability," IEEE Trans Power Syst, vol. 32, no. 2, pp. 1050-1063, March2017.

9. A. Chiş, J. Lundén and V. Koivunen, "Scheduling of plug-in electric vehicle battery charging with price prediction," IEEE PES ISGT Europe 2013, pp. 1-5, Lyngby, 2013.

10. I. Momber and T. Gómez, "The effect of mobility forecasts for stochastic charge scheduling of aggregated PEV," IEEE PES ISGT Europe 2013, pp. 1-5, Lyngby, 2013.

11. Arif, A. I., Ahamed, T. P. I., \& Al-Ammar, E. A. (2013). Pursuit algorithm for scheduling PEV charging. In 4th International Conference on Power Engineering, Energy and Electrical Drives (pp. 915-920). Istanbul.

12. C. Le Floch, F. Belletti, S. Saxena, A. M. Bayen and S. Moura, "Distributed optimal charging of electric vehicles for demand response and load shaping," 2015 54th IEEE Conference on Decision and Control (CDC), pp. 6570-6576, Osaka, 2015.

13. A. Chiş, J. Lundén and V. Koivunen, "Optimization of plug-in electric vehicle charging with forecasted price," 2015 IEEE International Conference on Acoustics, Speech and Signal Processing (ICASSP), pp. 2086-2089, South Brisbane, QLD, 2015

14. González Vayá, M., \& Andersson, G. (April 2016). Self-scheduling of plug-in electric vehicle aggregator to provide balancing services for wind power. IEEE Transactions on Sustainable Energy, 7(2), 886-899.

15. Delfino, F., Minciardi, R., Pampararo, F., \& Robba, M. (July 2014). A multilevel approach for the optimal control of distributed energy resources and storage. IEEE Transactions on Smart Grid, 5(4), 2155-2162.

16. Foster, J. M., Trevino, G., Kuss, M., \& Caramanis, M. C. (Dec. 2013). Plug-in electric vehicle and voltage support for distributed solar: Theory and application. IEEE Syst J, 7(4), 881-888.

17. R. Arulbel Benela and K. Jamuna, "Design of charging unit for electric vehicles using solar power", 2013 International Conference on Information Communication and Embedded Systems (ICICES), pp. 919-924, Chennai, 2013.

18. Ismail, M., Bayram, I. S., Abdallah, M., Serpedin, E., \& Qaraqe, K. (2015). Optimal planning of fast PEV charging facilities. March: IEEE Smart Grid and Renewable Energy.

19. S. Sun, Q Yang, W. Yan, "A novel Markov-based temporal-SoC analysis for characterizing PEV charging demand," IEEE Transactions on Industrial Informatics, no.99, pp.1-1, doi: https://doi.org/10.1109/TII.2017.2720694, 2017 
20. S. Sun, Q. Yang and W. Yan, "A novel statistical Markov-based approach for modeling charging demand of plug-in electric vehicles," 2016 China International Conference on Electricity Distribution (CICED), pp. 1-6, Xi'an, 2016.

21. Ruan, B., Yang, Q., Fang, X., \& Yan, W. (2014). Demand response under realtime pricing for domestic energy system with DGs. In 2014 International Conference on Power System Technology (pp. 3103-3110). Chengdu.

22. Li, G., \& Zhang, X. P. (March 2012). Modeling of plug-in hybrid electric vehicle charging demand in probabilistic power flow calculations. IEEE Transactions on Smart Grid, 3(1), 492-499.

23. Transport Research \& Innovation Portal, "Mobile Energy Resources in Grids of Electricity", Available [Online]: http://www.transport-research.info/sites/ default/files/project/documents/20140203_154622_76425_Deliverable_2.1_ Modelling_Electric_Storage_devices_for_Electric_Vehicles.pdf.

24. Romero, R., Franco, J. F. \& Leão, F. B. (Mar. 2016). A new mathematical model for the restoration problem in balanced radial distribution systems. IEEE Trans Power Syst, 31(2), 1259-1268.

25. .L. Feng, "Electric vehicle charging station planning", Ph.D. dissertation, Tianjin University, Tianjin, China, 2013.

26. The Wind Power, Available [Online]: http://www.thewindpower.net/.

27. The California Energy Almanac, Available [Online]: http://energyalmanac.ca. gov/renewables/solar/PV.html.

28. AMEO, Available [Online]: http://www.ameo.com.au/Electricity/Data/Priceand-Demand.

29. U.S. Department of Transportation Federal Highway Administration, SUMMARY OF TRAVEL TRENDS: 2009 National Household Travel Survey, Available [Online]: http://nhts.ornl.gov/2009/pub/stt.pdf.

\section{Submit your manuscript to a SpringerOpen ${ }^{\circ}$ journal and benefit from:}

- Convenient online submission

- Rigorous peer review

- Open access: articles freely available online

- High visibility within the field

- Retaining the copyright to your article

Submit your next manuscript at $\gg$ springeropen.com 